\title{
Strontium in the era of Gaia and LAMOST
}

\author{
Camilla J. Hansen ${ }^{1}$, Elisabetta Caffau ${ }^{1}$, and Maria Bergemann ${ }^{2}$ \\ ${ }^{1}$ Landerssternwarte, Heidelberg University \\ email: cjhansen@lsw.uni-heidelberg.de, ecaffau@lsw.uni-heidelberg.de \\ ${ }^{2}$ MPA Garching \\ email: mbergema@mpa-garching.mpg.de
}

\begin{abstract}
The formation and evolution of the heavy neutron-capture elements $(\mathrm{Z}>37)$ are to date not well understood. Therefore, abundance and galactic chemical evolution (GCE) studies of these heavy elements may carry key information to this open question. Strontium ( $\mathrm{Sr}$ ) is one of the two heavy elements ( $\mathrm{Sr}$ and $\mathrm{Ba}$ ) that show intrinsically very strong absorption lines even in extremely metal-poor stars (and remains detectable at low spectral resolution). Hence, the $4077 \AA$ Sr II line provides a unique insight into the behaviour of heavy neutron-capture elements at all metallicities and resolutions. Here the focus is on strontium, its 3D and NLTE (non-local thermodynamic equilibrium) corrections, as well as chemical evolution.
\end{abstract}

Keywords. Galaxy: halo, stellar content, stars: abundance

\section{Implications}

The NLTE corrections have been calculated using the model atom described in Bergemann et al. (2012). The majority of the Galactic chemical evolution (GCE) studies are only concerned with Sr II (due to the strong $4077.7 \AA$ Sr II line). We find that the GCE results based on LTE abundances of the Sr II resonance line are accurate, and can be used without large biases down to $[\mathrm{Fe} / \mathrm{H}] \sim-1.5$ (if NLTE corrections are not available). As seen from Table 1, the largest NLTE corrections are found in metal-poor giants if $[\mathrm{Fe} / \mathrm{H}]$ $<-2$. For comparison the 3D corrections are of similar size as the NLTE corrections, but of opposite sign. Convection can only be approximately treated in 1D (through mixing length theory), hence 1D and 3D differ due to treatment of convection, microturbulence, and the $3 \mathrm{D}$ corrections are sensitive to the line strength, the lower energy level of the line transition, and the element in question. Therefore, the 3D and NLTE correction will not always cancel. Further details on data analysis and galactic chemical evolution of $\mathrm{Sr}$ (both LTE and NLTE) can be found in Hansen et al. (2013).

Table 1. Corrections to $\mathrm{Sr}$

\begin{tabular}{|c|c|c|c|c|}
\hline SrII $(4077 \AA ̊)$ & $\Delta$ NLTE $($ SrII) & 3D (SrII) & $\operatorname{SrI}(4607 \AA)$ & $\triangle$ NLTE $(\mathrm{SrI})$ \\
\hline Dwarf $(\mathrm{EW} \sim 100 \mathrm{~m} \AA)$ & -0.08 & 0.08 & Dwarf $(\mathrm{EW} \sim 5 \mathrm{~m} \AA)$ & 0.34 \\
\hline Giant $(\mathrm{EW} \sim 160 \mathrm{~m} \AA)$ & -0.14 & 0.20 & Giant $(\mathrm{EW} \sim 35 \mathrm{~m} \AA)$ & 0.34 \\
\hline Reason for LTE/1D deviation & Strong line scattering & Convection & - & Over-ionisation \\
\hline Codes used & DETAIL & $\mathrm{CO}^{5} \mathrm{BOLD}$ & - & DETAIL \\
\hline
\end{tabular}

Notes: $E W=$ Equivalent Width of a dwarf $(T / \operatorname{logg} /[\mathrm{Fe} / \mathrm{H}] / \xi: 5900 / 4 /-2 / 1.5)$ and a giant (4500/2/-3/1.8).

\section{References}

Bergemann, M., Hansen, C. J., Ruchti, G., et al 2012, A\&A, 546, 90

Hansen, C. J., Bergemann, M., Cescutti, G., et al 2013, A\&A A, 551, 57 